\title{
DIVERSIDAD DE LA SUBFAMILIA CAMPOPLEGINAE - ICHNEUMONIDAE (HYMENOPTERA) EN LA CUENCA DEL RÍO CAÑETE - LUNAHUANÁ, PERÚ
}

\section{THE CAMPOPLEGINAE (HYMENOPTERA, ICHNEUMONIDAE) WASP SUBFAMILY, IN THE CAÑETE - LUNAHUANÁ RIVER VALLEY, PERU}

\author{
Alexander Rodríguez Berrío y Silvia Gutiérrez Bustamante ${ }^{2}$
}

\begin{abstract}
Resumen
El presente estudio se ha efectuado para determinar la diversidad de esta subfamilia en cuatro pisos altitudinales de la cuenca del río de Cañete-Lunahuaná, Provincia de Lima, Perú: La Encañada (200 m), San Jerónimo (400 m), Pacarán (600 m) y Zúñiga (800 m); durante el periodo de evaluación de un año. Los muestreos se efectuaron en el entorno de campos de cultivo a pequeña escala y el mecanismo de muestreo fue a través de la instalación de trampas del tipo Malaise, las que fueron ubicadas en vegetación silvestre. Los resultados muestran diversidad de Campopleginae (941 especímenes), determinándose los géneros Microcharops, Campoletis, Venturia, Campoplex, Prochas, Casinaria y Diadegma. La mayor riqueza de especies correspondió a La Encañada (23 morfoespecies) y la mayor abundancia de especímenes en San Jerónimo (362 individuos). El género Microcharops representa el $75 \%$ de la fauna de Campopleginae del área de estudio, siendo el género más dominante, le siguen en importancia Campoletis con $8.9 \%$ y Venturia $8.7 \%$. Con respecto a la proporción de sexos se determinó una mayor proporción de machos 1.48:1.00 con respecto a las hembras. Se registra por primera vez para el Perú a las especies $M$. taiticus y $M$. niger, siendo la primera la de mayor variabilidad y representada con cuatro morfotipos.
\end{abstract}

Palabras clave: Campopleginae, diversidad, gradiente altitudinal.

\begin{abstract}
The study was performed during one calendar year to determine the diversity of the Campopleginae wasp subfamily, present in the following four localities with different altitudes, along the Cañete - Lunahuana valley, Province of Lima, Peru: Encañada (200 m), San Jerónimo (400 m), Pacarán (600 m), and Zúñiga (800 m). The samples were collected in the vicinity of cultivated fields by using Malaise traps, placed above wild shrubs and herbs. 941 specimens in the Subfamily were collected belonging to the following genera: Campoletis, Campoplex, Casinaria, Diadegma, Microcharops, Prochas, and Venturia. The highest species richness level was found at Encañada (23 morphospecies), and the highest number of collected specimens were obtained at San Jerónimo (362 individuals). Microcharops represent $75 \%$ of the Campopleginae fauna found in the studied area, being the most dominant genus; followed in importance by Campoletis with $8.9 \%$, and Venturia with $8.7 \%$. With respect to the sex ratio 1.48 more males than females were trapped. For the first time Microcharops taiticus and Microcharops niger were recorded for Peru; the first species being more variable (with four morphotypes) than the second one.
\end{abstract}

Key words: Campopleginae, genera, Peru, diversity, altitudinal gradient.

\section{Introducción.}

La Cuenca del Río Cañete-Lunahuaná, presenta una fuerte gradiente altitudinal que se origina desde el deshielo de los glaciares del nevado Ticlla, en la vertiente occidental de los Andes Centrales del Perú a $4830 \mathrm{~m}$., hasta su desembocadura en el Océano Pacífico (Acosta, 2009).

Los insectos ejercen un gran impacto en los ecosistemas terrestres, éstos, son importantes por su papel en el funcionamiento de ecosistemas naturales actuando como predadores, parasitoides, fitófagos, saprófagos o polinizadores (Odony, 2005). Los parasitoides son considerados como bioindicadores, porque representan la diversidad de los hospederos que atacan y también son de utilidad en trabajos de conservación, por ser sensibles a perturbaciones ecológicas especialmente por pesticidas (Sharkey, 2007).

La fauna de Ichneumonidae en Perú, tiene escasos estudios a pesar de ser posiblemente de una gran diversidad. En una publicación reciente, Rodríguez Berrio et al. (2009), listan 391 especies, de las cuales 
139 son endémicas y exclusivas de Perú (Alvarado, 2011).

Los Campopleginae, son conocidos por su importancia en el control de poblaciones de insectos plaga, especialmente Campoletis spp., ha sido recuperada frecuentemente en cultivos de maíz, algodón, frejol, entre otros (Alvarado, 2001; Huayta, 2013).

El trabajo tiene como objetivo el conocer la diversidad e importancia de la Subfamilia Campopleginae y como están distribuidos en una gradiente altitudinal de 200, 400, 600 y $800 \mathrm{~m}$.

\section{Materiales y métodos.}

El material biológico.

Entre los años 2009 al 2010, se efectuaron 33 muestreos a lo largo de la Cuenca del río CañeteLunahuaná, donde fueron instaladas 08 trampas del tipo Malaise, en una gradiente altitudinal de 200, 400, 600 y 800 m (Tabla 1$)$.

Tabla1: Coordenadas geo-referenciales de ubicación de las trampas en la Cuenca del río Cañete-Lunahuaná.

\begin{tabular}{cccc}
\hline $\begin{array}{c}\text { Altura } \\
\text { teórica }\end{array}$ & Localidad & Trampa & Coordenadas \\
\hline $200 \mathrm{~m}$. & La Encañada & Trampa 1 & S: $13^{\circ} 05.203^{\prime}$ Wo: $76^{\circ} 14.876^{\prime}$ \\
& & Trampa 2 & S: $13^{\circ} 05.203^{\prime}$ Wo: $76^{\circ} 14.876^{\prime}$ \\
$400 \mathrm{~m}$. & San Jerónimo & Trampa 3 & S: $13^{\circ} 00.022^{\prime}$ Wo: $76^{\circ} 09.489^{\prime}$ \\
& & Trampa 4 & S: $13^{\circ} 00.025^{\prime}$ Wo: $76^{\circ} 09.480^{\prime}$ \\
$600 \mathrm{~m}$. & Pacarán & Trampa 5 & S: $12^{\circ} 54.525^{\prime}$ Wo: $76^{\circ} 05.549^{\prime}$ \\
& & Trampa 6 & S: $12^{\circ} 54.561^{\prime}$ Wo: $76^{\circ} 05.593^{\prime}$ \\
$800 \mathrm{~m}$. & \multirow{2}{*}{ Zúñiga } & Trampa 7 & S: $12^{\circ} 51.667^{\prime}$ Wo: $76^{\circ} 01.867^{\prime}$ \\
& & Trampa 8 & S: $12^{\circ} 51.671^{\prime}$ Wo: $76^{\circ} 01.890^{\prime}$ \\
\hline
\end{tabular}

La colecta de los especímenes de los sectores evaluados, se efectuó con una frecuencia de 15 días, de la siguiente forma: la primera evaluación quincenal, en forma descendente, y la siguiente evaluación, fue de forma ascendente, así sucesivamente. Las muestras fueron preservadas en alcohol al $70 \%$ en frascos debidamente etiquetados con los datos de colecta y remitidas al laboratorio de Hymenoptera del Museo de Entomología "Klaus Raven Büller.” de la Universidad Nacional Agraria La Molina, donde los ejemplares fueron clasificados a nivel de la subfamilia Campopleginae (Hymenoptera: Ichneumonidae).

Para la determinación de los géneros de los especímenes de Campopleginae, se utilizó la terminología y el criterio taxonómico de Gauld (1991) y Townes (1970b), estableciendo morfoespecies, en caso de encontrarse diferencias morfológicas. Sólo el género Microcharops Roman, 1910 se trabajó a nivel específico (Gupta, 1987), en caso de encontrar diferencias y variaciones en coloración se determinaron morfotipos. Las especies fueron confirmadas por la Dra. Angélica Penteado de la Universidade Federal São Carlos (UFSCar- Brasil). El material determinado fue depositado en la colección entomológica del Museo "Klaus Raven Büller”.

Descripción de la zona de estudio.

Sector de “La Encañada”: Las trampas fueron instaladas a $250 \mathrm{~m}$ de distancia al río y a una altitud de 200 m. La composición vegetal correspondió a una vegetación silvestre, entre las que se destacan: caña brava (Gynerium sagittatum), chilca (Baccharis sp), quinua silvestre (Chenopodium album), cola de caballo (Equisetum arvense), cerraja (Sonchus oleraceus), pájaro bobo (Tessaria integrifolia), paico (Chenopodium ambrosoides) entre otros, como también a cultivos agrícolas a pequeña escala: Zea mays, Phaseolus vulgaris, Pisum sativum, Ipomoea batatas, Manihot esculenta y frutales como Vitis vinífera, Persea americana y Mangifera indica, este hábitat corresponde a la parte geográfica donde la cuenca se abre y comienza a extenderse. En el valle de cañete en la época de lluvias (noviembre a febrero) la napa freática es casi superficial creando un ambiente pantanoso.

Sector de "San Jerónimo": Las trampas fueron instaladas a $70 \mathrm{~m}$ de distancia al río y a una altitud de $400 \mathrm{~m}$. La composición vegetal del entorno de la trampa fue un vergel de plantas de frutales: Annona cherimola, Ficus carica, Pyrus malus, Inga feuillei, Perse americana, Eriobotrya japónica, Vitis vinífera; también destacan cultivos de pequeñas parcelas agrícolas de Zea mays y Phaseolus lunatus, así como de plantas silvestres: gramalote (Panicum purpurasces), amor seco (Bidens pilosa), diente de león (Taraxacum officinale), yuyo hembra (Amaranthus sp.) y Crotalaria sp., entre las más frecuentes.

Sector de "Pacarán”: Las trampas fueron instaladas a $600 \mathrm{~m}$ de distancia al río y a una altitud de $600 \mathrm{~m}$. En este sector, las trampas se instalaron en la parte más alta de la cuenca muy cerca al cerro, por razones de seguridad. La vegetación estaba compuesta por plantas silvestres como: Crotalarias sp., Bidens pilosa, Asclepias curassavica, Taraxacum officinale y cultivos agrícolas a pequeña escala de Medicago sativa, Zea mays y Phaseolus lunatus, también se observó frutales que conformaban un vergel de: Pyrus malus, Eriobotrya japónica, Cydonia oblonga, Prunus domestica, Inga feuillei, Vitis vinífera, Opuntia ficusindica; así como la presencia de árboles de álamo (Populus sp.) y huarango (Prosopis sp.).

Sector de “Zuñiga”: Las trampas fueron instaladas a $200 \mathrm{~m}$ de distancia al río y a una altitud de 800 m. La composición vegetal donde se colocaron las trampas, fue de plantas silvestres: campanilla (Convolvulus sp.), Crotalarias sp., Bidens pilosa y Amaranthus sp. También una plantación vergel de Persea americana, Prunus pérsica y un número 
menor de plantas de Passiflora edulis, Mangifera indica, Vitis vinífera, Carica papaya, Inga feuillei, Citrus reticulata, Citrus sinensis. Así como parcelas de cultivos agrícolas de Phaseolus lunatus y Phaseolus vulgaris.

Estimadores de diversidad.

Los datos obtenidos por la naturaleza del trabajo no corresponden a una distribución normal, por ello se utilizó estadísticos no paramétricos y estimadores de diversidad; mediante curvas de acumulación de especies, "Estimates 750" (Cowell \& Coddington, 1994; Cowell, 2006); índices de similaridad BrayCurtis, “BioDiversyti" (McAleece, 1997), para determinar la diversidad alfa, riqueza específica y diversidad beta en cada gradiente altitudinal.

Características climáticas.

La quebrada del valle de Cañete - Lunahuaná tiene un clima muy templado con condiciones climáticas homogéneas entre los sectores de San Jerónimo, Lunahuana hasta Zúñiga donde en el invierno hay radiación solar constate, con temperaturas medias de $14-21^{\circ} \mathrm{C}$, llegando en verano hasta más de $30^{\circ} \mathrm{C}$; esta difiere del sector de la Encañada y de la parte baja donde se extiende el valle de Cañete, con condiciones de baja nubosidad, sin brillo solar, alta humedad relativa durante el invierno y con temperaturas medias de $13^{\circ} \mathrm{C}$.

\section{Resultados y discusión.}

Se determinaron siete géneros: Campoplex Förster, 1869 (N=34); Prochas Walkley, 1959 (N=23); Campoletis Förster, 1869 (N=84); Venturia Schrottky, 1902 (N=82); Casinaria Holmgren, 1859 (N=6); Diadegma Förster, 1869 (N=4) y Microcharops Roman, 1910 (N=708 y S=03). Los mismos están distribuidos en una proporción de 60\% machos y 40\% hembras.

Existe una amplia variación en la proporción sexual de los especímenes, que podría atribuirse al sistema de diferenciación sexual (Haplodiploidia), también a condiciones externas, tamaño de hospedero, temperatura, fotoperiodo y factores nutricionales (Da Silva, 2012).

En Microcharops se han determinado las siguientes especies:

Microcharops taiticus (Holmgren, 1868).

La especie más frecuente con más del 70\% de abundancia de todos los Campopleginae del área de estudio ( $\mathrm{N}=469$ ). Se ha agrupado en cuatro morfotipos debido a la variación del patrón de coloración y la forma como se presenta en algunas estructuras morfológicas: Cabeza: presencia de matices oscuros en el borde posterior del escapo y pedicelo. Mesosoma: presencia de marcas negras en el margen interno del fémur anterior y de infuscaciones oscuras basal en los fémures medios. Variación de intensidad de color de los fémures posteriores. Metasoma: variación del color negro del tergito 2 y 3. También la intensidad y forma de líneas amarillentas dorsalmente presentes, a partir del tercer tergito los matices oscuros se proyectan más allá de la línea media.

Las descripciones de las especies del género Microcharops por Gupta (1987), no son muy precisas en referencia a los aspectos cualitativos de color en algunas estructuras morfológicas. Es así que algunas especies como $M$. taiticus, muestran variación en el color de sus trocánteres posteriores; en el tergo 2 la intensidad del color de una línea amarillenta y la presencia de marcas negruzcas en el tergo 3; sin precisar su forma y el grado que abarca en el tergo. En referencia a la ubicación de marcas negruzcas en la pata media, no esclarecen si es en el fémur o tibia. También se precisa que las especies examinadas por Gupta (1987), no corresponden a ninguna registrada para el Perú, y quizás es factible la variación encontrada.

Microcharops tibialis (Cresson, 1872).

Especie también frecuentemente hallada en el área de estudio ( $\mathrm{N}=236)$. Por las variaciones de patrones de coloración fueron clasificados en cuatromorfotipos: Cabeza: presencia de una línea oscura en el borde lateral externo del escapo y pedicelo, flagelo con el borde anterior marrón claro y marrón oscuro en el borde posterior. Mesosoma: Variación del color de los fémures anterior y medio de marrón claro a marrón amarillento, en este último se observa pigmentaciones oscuras a muy oscuras en la parte basal como también en toda su longitud. Los fémures posteriores marrón anaranjado a oscuros. Trocantes posteriores amarillentos apicalmente, infuscadas de color marrón en su base y su trocantellus amarillo. Metasoma: Tergito 2, de color oscuro, con una banda estrecha subapical amarilla.

La descripción de $M$. tibialis por Gupta (1987), también es ambigua en referencia a la presencia de marcas parduzcas en los fémures anterior y medio, los cuales no precisan el lugar de estos. Así mismo se identificó la existencia de la gama del color parduzco en los fémures posteriores, esto se observó en algunos especímenes que eran de color anaranjado - café y en otros está combinado dicho color. El término "generalmente" no es preciso, con respecto al color negro del abdomen.

Microcharops níger Gupta, 1987

Especie ocasionalmente encontrada $(\mathrm{N}=3)$.

Escapo y pedicelo marrón amarillento en su borde anterior y su borde posterior con manchas oscuras. Flagelo en el borde anterior marrón claro y en borde posterior marrón oscuro. Patas anteriores marrón amarillento, trocánter y trocantellus amarillento. Coxas negras. Fémur medio marrón amarillento con marcas negras basalmente, trocánter y trocantellus amarillento. Fémur posterior color negruzco, trocánter negro en toda su extensión (trocánter amarillento apicalmente, matizado de color negro en su base) y trocantellus amarillento. Metasoma negro. 
Riqueza Específica por localidades.

La Encañada $(200 \mathrm{~m})$ fue la más diversa al registrar todos los géneros $\mathrm{y}$ también la de mayor riqueza (23 morfoespecies); seguido de Zuñiga (800 m) con 20 morfoespecies; sin embargo, el sector de San Jerónimo (400 m) registró la mayor captura de individuos $(\mathrm{N}=362)$. Resultados similares fueron encontrados al trabajar con la familia Braconidae por Huayta (2013), quien determinó que en la Encañada se registró la mayor riqueza con 12 especies de la subfamilia Microgastrinae y el sector de San Jerónimo la mayor abundancia de individuos ( $\mathrm{N}=387)$ de la Familia Braconidae, en la misma área de estudio.

Los géneros Microcharops, Campoletis, Venturia y Prochas, muestran una amplia distribución altitudinal; mientras que Campoplex, Casinaria y Diadegma, son predominantes en altitudes bajas (200 $\mathrm{m})$, en tanto que Campoletis fue abundante a $800 \mathrm{~m}$ (Tabla 2); este resultado es similar al obtenido por Penteado-Dias \& Odony (2004) y Loibel et al. (2010), quienes señalan que Campoletis es más abundante a altitudes entre 856-1600 m respectivamente.

Distribución estacional.

En el verano la densidad poblacional se incrementa en todos los géneros ( $\mathrm{N}=428$ ) representando un 45\% de la población total. Puede considerarse que la estación de verano y otoño, son las de mayor abundancia en comparación con otras estaciones, como se detalla en la (Tabla 3). Según Alvarado (2011), la fenología de las poblaciones de Ichneumonidae, en Cañete presenta cierta estacionalidad, siendo las estaciones de primavera y verano, donde se incrementan las poblaciones, alcanzando las máximas durante el verano, este es similar al obtenido durante el estudio.

Los géneros Microcharops, Campoletis $y$ Campoplex, son de amplia distribución estacional durante todo el año, con predominancia de Microcharops, que registró 708 individuos, el género Casinaria se registra sólo en verano y otoño (Tabla 3); sin embargo, para Penteado-Dias \& Odony (2004), Casinaria fue colectada tanto en época de lluvia

Tabla 2: Distribución de los géneros de Campopleginae en la Cuenca del río Cañete-Lunahuaná.

\begin{tabular}{|c|c|c|c|c|c|}
\hline Géneros & $\begin{array}{l}\text { La } \\
\text { Encañada }\end{array}$ & $\begin{array}{l}\text { San } \\
\text { Jerónimo }\end{array}$ & Pacarán & Zúñiga & Total \\
\hline Microcharops & 51 & 323 & 98 & 236 & 708 \\
\hline Campoletis & 15 & 17 & 12 & 40 & 84 \\
\hline Venturia & 56 & 12 & 9 & 5 & 82 \\
\hline Campoplex & 23 & 1 & 5 & 5 & 34 \\
\hline Prochas & 4 & 8 & 7 & 4 & 23 \\
\hline Casinaria & 3 & 1 & 1 & 1 & 6 \\
\hline Diadegma & 4 & 0 & 0 & 0 & 4 \\
\hline$(\mathrm{N})$ & 156 & 362 & 132 & 291 & 941 \\
\hline$(\mathrm{S})$ & 23 & 15 & 18 & 22 & \\
\hline
\end{tabular}

(Noviembre-Abril) como época seca (Junio-Octubre). Diversidad.

El género Mirocharops presentó la mayor abundancia con 708 especímenes, que representan el $75 \%$ de la fauna de Campopleginae, en tres especies $M$. tibialis, $M$. niger y $M$. taiticus; esta última con más de $66 \%$ de abundancia. Estos resultados demuestran la importancia de este género, que se encuentra bien distribuido en todos los hábitats estudiados desde los $200 \mathrm{~m}$ hasta los $800 \mathrm{~m}$. Las especies Microcharops tibialis y Microcharops taiticus, son nuevos registros para el Perú.

Curvas de acumulación de especies.

Se analizó la riqueza obtenida en el área de estudio mediante curvas de acumulación de especies, mediante la interpolación de los datos de abundancia y riqueza, utilizando el estadístico no paramétrico de Estimates 750 (Cowell, 2006), los resultados demuestran que el esfuerzo alcanzado supera el $81 \%$ de eficiencia de muestreo (Figura 1).

Huayta (2013), estudiando la diversidad de Braconidae en la misma zona de estudio determinó una eficiencia de muestreo de $82 \%$ y $91 \%$ para la subfamilia Microgastrinae, resultados que son muy similares al obtenido en el presente estudio. Por otro lado, en estudios de la fauna de Ichneumonidae de ecosistemas de costa del Perú, Alvarado (2011) obtuvo una eficiencia similar al $80 \%$ utilizando trampas Malaise, para las cuencas de Cañete y Huaral. Estructura de la comunidad.

La estructura de la comunidad de Campopleginae medidos a través de índices de diversidad muestran que el índice de equitatividad de Shannon - Weaver $(\mathrm{H})$ en promedio para la cuenca del río de Cañete-Lunahuaná, corresponde a 2.12, lo que indica una estructura de la comunidad intermedia, es decir las diferentes especies no tienen una distribución equitativa; así lo demuestra el índice de dominancia de Simpson con 4.3. Esta falta de equitatividad y dominancia está determinada por el género Microcharops con más de $75 \%$ de la abundancia, la misma que se concentra en una sola especie $M$. taiticus 


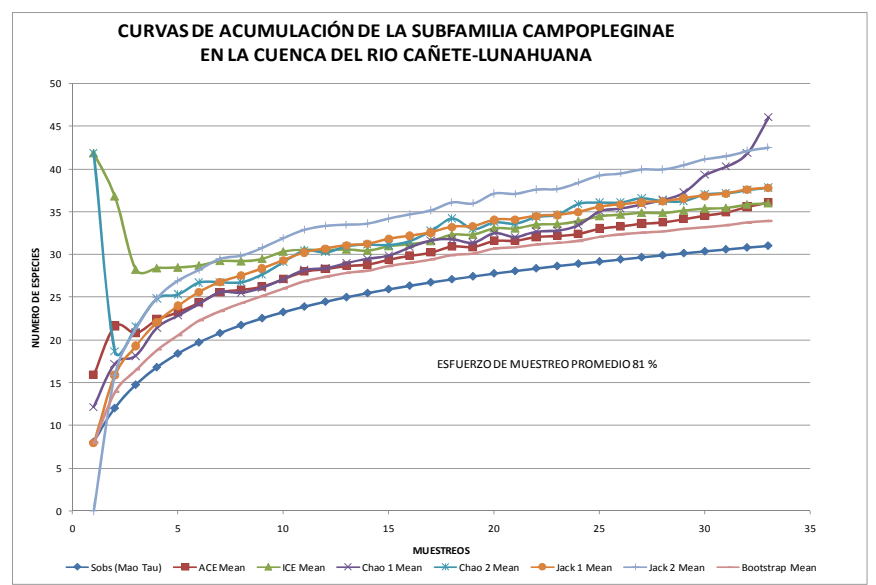

Figura 1. Gráfico de curvas de acumulación y esfuerzo de muestreo.

\begin{tabular}{rccccccc} 
Muestreo & ACE & ICE & Chao1 & Chao2 & Jack1 & Jack2 & Bootstrap \\
\hline 31 & 36 & 36 & 46 & 38 & 38 & 42 & 34 \\
100 & 86 & 86 & 67 & 82 & 82 & 73 & 91 \\
Promedio & $81 \%$ & & & & & & \\
\hline
\end{tabular}

que representa más del $60 \%$ de la población de Campopleginae del área de estudio.

El índice de Shannon fue mayor en la Encañada con 0.4678, esto implica que todos los géneros están representados y distribuidos con cierta equitatividad, a diferencia del sector de San Jerónimo el cual presenta el mayor índice de dominancia de Simpson con 0.9905, y se explica por la alta abundancia de los géneros Microcharops, Campoletis y Venturia, que fueron los grupos predominantes.

İndices de similaridad.

La estructura de la comunidad de Campopleginae en los diversos pisos altitudinales es muy variable, en conjunto todos los habitats estudiados mantienen más del 50\% de especies compartidas; siendo los hábitats de San Jerónimo (400 m) y Zúñiga (800 m) los que presentan un $76 \%$ de similaridad, en tanto que los hábitats que presentan un menor número de especies compartidas corresponden a La Encañada (200 m) y Pacarán (600 m) (Figura 2). Según Loibel et al. (2010) existe una mayor similaridad en las huertas de Araraquara $(664 \mathrm{~m})$, como probable resultado de la abundancia de Microcharops, existiendo una similitud con el resultado obtenido en la investigación, que muestra un $60 \%$ de abundancia en altitudes de $600 \mathrm{~m}$.

Analizando la composición de especies por estaciones del año se ha determinado una alta similaridad, para la comunidad de Campopleginae, entre el verano y otoño con un $73 \%$, probablemente debido a la riqueza de especies que comparten entre las dos estaciones (07 géneros) y entre el invierno y primavera un 67\%, por la presencia de sólo tres géneros (Figura 3). Está composición en particular estaría determinada por las condiciones ambientales propias de la quebrada y por el rol biológico que cumplen los cultivos a pequeña escala.

Las condiciones de la quebrada de Lunahuaná son muy homogéneas en condiciones climáticas, especialmente desde el sector de San Jerónimo hasta Zúñiga donde en el invierno hay radiación solar constate, esta difiere del sector de la Encañada y del valle con condiciones de baja nubosidad, sin brillo solar y alta humedad relativa que influye en las bajas poblaciones de Campopleginae registradas entre las estaciones de invierno y primavera, es por este motivo que se obtuvieron datos diferentes según Alvarado (2011), que manifiesta que durante las estaciones de primavera y verano se incrementan las poblaciones de Ichneumonidae en la localidad de Cañete.

\section{Conclusiones.}

La Subfamilia Campopleginae en la quebrada del río Cañete-Lunahuaná, está representada por siete géneros: Diadegma, Venturia, Campoplex, Casinaria, Campoletis, Prochas y Microcharops.

Se determinaron tres especies Microcharops taiticus, Microcharops tibialis y Microcharops niger.

El porcentaje de abundancia de los géneros en la población total fue: Microcharops 75\%, Campoletis 8.9\%, Venturia 8.7\%, Campoplex 3.6\%, Prochas $2.4 \%$, Casinaria $0.6 \%$ y Diadegma $0.4 \%$.

Se registra por primera vez para el Perú a las especies $M$. taiticus y $M$. niger, siendo la primera la de mayor variabilidad con cuatro morfotipos.

La distribución estacional de Campopleginae se concentra entre los meses de verano-otoño y disminuye en el invierno y primavera. La composición de especies también guarda relación entre las 


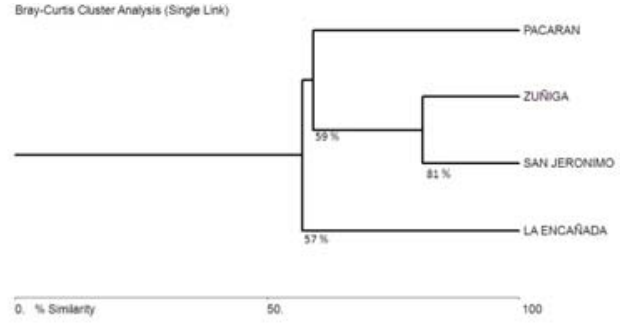

Figura 2. Dendograma de Similaridad entre localidades en la Cuenca del río CañeteLunahuaná.

Leyenda: La Encañada 200 m; San Jerónimo 400 m; Pacarán 600 m y Zúñiga 800 m.
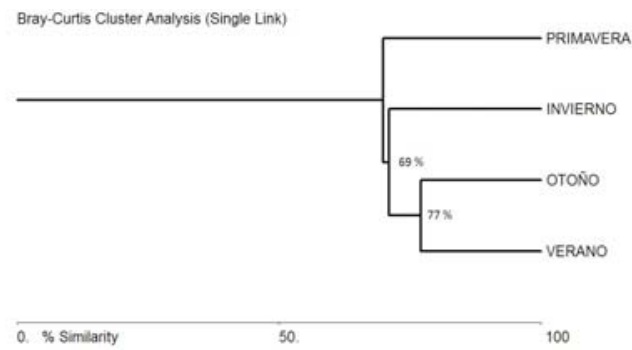

Figura 3. Dendograma de Similaridad entre estaciones del año, en la cuenca del río CañeteLunahuaná.

estaciones obteniéndose la mayor similaridad entre el verano y otoño con $73 \%$, frente a un $67 \%$ entre primavera e invierno.

\section{Agradecimientos.}

El presente trabajo se realizó con financiamiento del Proyecto Ichneumonidae del Museo "Klaus Raven Büller”, de la Universidad Nacional Agraria La Molina (Perú).

\section{Literatura citada.}

Acosta R. 2009. Estudio de la cuenca alto andina del río Cañete (Perú): Distribución altitudinal de la comunidad de macroinvertebrados bentónicos y caracterización hidroquímica de sus cabeceras cársticas. Tesis para optar el Título de Doctor. Universidad de Barcelona. 133 p.

Alvarado M. 2011. Biodiversidad de Ichneumonidae (Hymenoptera) en tres hábitats de ecosistemas de Costa, Sierra y Selva de Perú. Tesis para optar el grado de Magister Scientiae en Entomología. Universidad Nacional Agraria La Molina. 162 p.
Colwell R.K \& Coddington J.A. 1994. Estimating terrestrial biodiversity through extrapolation. Philos. Trans. R. Soc. London. 345: $101-118$.

Colwell R.K. 2006. EstimateS: Statistical estimation of species richness and shared species from samples. Version 8. Persistent URL <purl.oclc.org/estimates>

Da Silva A.P. 2012. Estudo da Fauna de Pimplinae (Hymenoptera: Ichneumonidae) em áreas de cerrado no estado São Paulo. Tesis (Doutorado). São Carlos Brasil. Universidade Federal de São Carlos. 123 p.

Fernández F. \& Sharkey M.J. 2006. Introducción a los Hymenoptera de la Región Neotropical. Sociedad Colombiana de Entomología y Universidad Nacional de Colombia, Bogotá D.C 894 pp.

Gauld I. 1991. The Ichneumonidae of Costa Rica 1, Introduction, keys to subfamilies, and keys to the species of the lower pimpliform subfamilies: Rhyssinae, Pimplinae, Poemeniinae, Acaenitinae and Cylloceriinae. Florida, EEUU. Mem. Amer. Entomol. Inst. 589 pp.

Gupta V. 1987. Contributions of the American Entomolgical Institute. A revisión of the Genus Microcharops (Hymenóptera: Ichneumonidae). Volume 23, Number 2, 1987.35 pp.

Hanson P.E. \& Gauld I.D. 2006. Hymenóptera del Trópico. Memoirs of the American Entomological Institute, Volume 77. $994 \mathrm{pp}$

Huayta F.E. 2013. Distribución Altitudinal de parasitoides de la familia Braconidae en la Cuenca del río Lunahuaná. Tesis (Ingeniero Agrónomo). Lima, Perú. Universidad Nacional Agraria La Molina, Facultad de Agronomía.144 p.

Loibel S.D., Odony H.C \& Penteado-Dias A. 2010. Fauna de Campopleginae (Hymenoptera, Ichneumonidae) em hortas orgânicas em Araraquara e São Carlos, SP, Brasil. 5 pp. Biota Neotrop. 10(2).

McAleece Neil. 1997. BioDiversityProffesional Beta, The Natural History Museum and The Scottish Association For Marine Science.

Odony H.C. 2005. Estudo dos Campopleginae (Hymenóptera, Ichneumonidae) Neotropicais com enfase na fauna da mata Atlantica, Brasil. Tesis (Mestrado). São Carlos - Brasil. Universidade Federal de São Carlos. 93 p.

Penteado-Dias A.M \& Odony H.C. 2004. Altitudinal and seasonal distribution of Campopleginae genera (Hymenoptera, Ichneumonidae) in the Atlantic rain forest, Campos do Jordão, SP.

Rodríguez-Berrío A., Bordera S. \& Sääksjärvi I.E. 2009. Checklist of Peruvian Ichneumonidae (Insecta, Hymenoptera). Zootaxa 2303:1-44 pp.

Sharkey M.J. 2007. Phylogeny and Classification of Hymenoptera. Zootaxa 1668:521-548.

Townes H.K. 1970b. The genera of Ichneumonidae, Part III. Mem. Amer. Ent. Inst.13(1969): 1-307.

${ }^{1}$ Universidad Nacional Agraria La Molina, Departamento Académico de Entomología, Av. La Molina s/n La Molina, Apartado 12-056, Lima, Perú. Correo electrónico arodriber@lamolina.edu.pe

2 Universidad Nacional Agraria La Molina, Departamento Académico de Entomología, Av. La Molina s/n La Molina, Apartado 12-056, Lima, Perú. Correo electrónico: apanteles720@gmail.com 